Psychotherapeut $2021 \cdot 66: 406-413$

https://doi.org/10.1007/s00278-021-00530-5

Angenommen: 16. Juli 2021

Online publiziert: 30. August 2021

(c) Der/die Autor(en) 2021

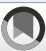

Check for
updates

\section{Einfluss akzeptanzfördernder Interventionen auf die Nutzung einer Online-Selbsthilfe}

\author{
Naturalistische Untersuchung in der (teil-)stationären \\ psychosomatischen Psychotherapie
}

\author{
Adina Kreis ${ }^{1}$ Jan Becker ${ }^{2}$. Manfred E. Beutel' · Rüdiger Zwerenz' \\ 'Klinik und Poliklinik für Psychosomatische Medizin und Psychotherapie, Universitätsmedizin der \\ Johannes Gutenberg-Universität, Mainz, Deutschland \\ ${ }^{2}$ Institut für Lehrergesundheit am Institut für Arbeits-, Sozial- und Umweltmedizin, Universitätsmedizin \\ der Johannes Gutenberg-Universität, Mainz, Deutschland
}

\section{In diesem Beitrag}

- Fragestellungen

- Studiendesign und Untersuchungsmethoden

Studiendesign - Akzeptanzfördernde Intervention - Datenerhebung • Datenauswertung

- Ergebnisse

Stichprobenbeschreibung - Akzep$\operatorname{tanz} \cdot$ Initiale Nutzung • Adhärenz • Auswirkung der Einstellung

- Diskussion

Ziel der Studie - Interpretation der Ergebnisse und Vergleich mit der Literatur • Limitationen und Stärken der Studie

Die Autoren A. Kreis und J. Becker teilen sich die Erstautor*innenschaft.

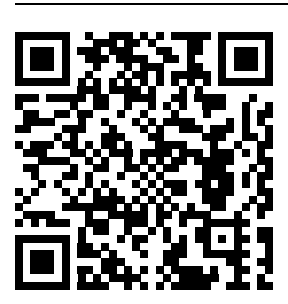

QR-Code scannen \& Beitrag online lesen
Zusammenfassung

Hintergrund: Trotz wachsender empirischer Evidenz zur Wirksamkeit in der Behandlung psychischer Erkrankungen sind internetbasierte Interventionen (IBI) mit geringen Akzeptanz- und hohen Abbruchraten konfrontiert. Akzeptanzfördernde Interventionen (AFI) stellen eine vielversprechende Maßnahme dar, um die Akzeptanz zukünftiger Nutzer*innen zu verbessern. Die Wirksamkeit von AFI auf die Adhärenz, die maßgeblichen Einfluss auf die Wirksamkeit von IBI hat, wurde bisher in nur wenigen empirischen Untersuchungen überprüft.

Ziel der Arbeit (Fragestellung): Ziel der vorliegenden Untersuchung ist die Überprüfung des Einflusses einer AFI auf die Akzeptanz, die initiale Nutzung und die Adhärenz des transdiagnostischen psychodynamischen Online-Selbsthilfeprogramms „Die Kraft der eigenen Emotionen nutzen (KEN-Online)".

Material und Methoden: Es wurden Routinedaten von 231 Patient*innen ausgewertet, die während ihrer (teil-)stationären Behandlung im Rahmen einer ins Routinesetting integrierten AFI das Angebot bekommen haben, KEN-Online behandlungsbegleitend zu nutzen. Im Kohortendesign werden Patient*innen, die an einer AFI teilgenommen haben, mit einer historischen Kontrollgruppe von Patient*innen verglichen, die dieses Angebot nicht erhielten. Zudem wird der Zusammenhang zwischen der Einstellung und der Akzeptanz des Programms in der Untersuchungsgruppe (mit AFI) überprüft. Ergebnisse: Sowohl die Akzeptanz als auch die initiale Nutzung von KEN-Online waren um knapp $20 \%$ höher (jeweils $p<0,01$ ) unter den Teilnehmenden der AFI-Gruppe, verglichen mit der Kontrollgruppe. In Bezug auf die Adhärenz von KEN-Online zeigte sich ein kleiner Unterschied dahingehend, dass Teilnehmende der Kontrollgruppe signifikant $(p<0,05)$ mehr Einheiten abgeschlossen haben als Teilnehmende der Interventionsgruppe. Allerdings war der Anteil der "completer" von KEN-Online mit $13,4 \%$ insgesamt sehr gering. Ein Einfluss der Einstellung auf die Akzeptanz wurde nicht nachgewiesen.

Diskussion: Die vorliegende Untersuchung zeigt, dass AFI dazu beitragen können, die Akzeptanz und initiale Nutzung von IBI zu fördern, indem Patient*innen der (teil-)stationären Routineversorgung standardisiert und strukturiert über die Intervention informiert werden. Hinsichtlich der Förderung der Adhärenz sind jedoch zusätzliche Maßnahmen erforderlich.

\section{Schlüsselwörter}

Internetbasierte Intervention · Akzeptanz · Behandlungsadhärenz · Einstellung · Inanspruchnahme der Gesundheitsversorgung 
Geringe Akzeptanz- und hohe Dropout-Raten stellen v.a. für die Implementierung von psychotherapeutischen Online-Interventionen in die Routineversorgung eine Herausforderung dar. Durch standardisierte und strukturierte Informationen von Patient*innen vor Beginn einer Intervention sollen potenzielle Nutzer*innen auf internetbasierte Interventionen (IBI) vorbereitet sowie ihre Akzeptanz und ihre Adhärenz gesteigert werden.

\section{Hintergrund}

Im Laufe ihrer Lebensspanne sind 4 von 10 Menschen in Deutschland von einer psychischen Erkrankung betroffen (Jacobi et al. 2014). Psychische Erkrankungen verlaufen oft chronisch, und im Vergleich zur Allgemeinbevölkerung sind die Lebenserwartung um 10 Jahre verkürzt und die Lebensqualität beeinträchtigt (Walker et al. 2015). Trotzdem erhalten nur $10-40 \%$ der Betroffenen eine adäquate leitliniengerechte Behandlung (Jacobi et al. 2014). Digitale Gesundheitsanwendungen für Menschen mit psychischen Erkrankungen entwickelten sich in den letzten Jahren zunehmend als vielversprechende Ergänzung zur Richtlinienpsychotherapie, um die bestehende Versorgungslücke zu verkleinern (Zwerenz und Beutel 2017). Die Wirksamkeit von IBI konnte in zahlreichen randomisierten kontrollierten Studien nachgewiesen werden, z.T. sind die Effekte sogar mit denen traditioneller „Face-to-face“(F2F)-Therapie vergleichbar (Andersson et al. 2014, 2019). Sie sind ortsunabhängig, zeitlich flexibel einsetzbar und können zudem eine kosteneffiziente Behandlungsmöglichkeit darstellen (Lin et al. 2013; Hedman et al. 2012). Als Präventions- und Nachsorgeelement sind sie ein wichtiger Bestandteil einer gestuften Gesundheitsversorgung (Lin et al. 2013). Jedoch zeigt sich bei der Implementierung in die Praxis oftmals, dass die Wirksamkeit von IBI geringer ist als in kontrollierten Studien, was insbesondere auf eine geringe Adhärenz und Motivation zur Nutzung zurückgeführt wurde (Gilbody et al. 2015). Somit stellt insbesondere die Implementierung von $\mathrm{IBI}$ in die Praxis eine Herausforderung dar, da es zwar mittlerweile viele IBI für Men- schen mit psychischen Erkrankungen gibt, der Anteil derjenigen, die diese Angebote tatsächlich nutzen, aber oftmals nur gering ist (Moessner und Bauer 2017). Gerade unter Psychotherapiepatient*innen bestehen im Vergleich zur Allgemeinbevölkerung stärkere Vorbehalte bezüglich des Datenschutzes und Sorgen bezüglich der Gefahr von Missverständnissen bei Behandlungsangeboten ohne direkten F2F-Kontakt (Gieselmann et al. 2015).

\section{Akzeptanz als Schlüssel zur Implementierung von internetbasierten Interventionen}

Trotz vielfältiger Wirksamkeitsbelege zu IBI identifizieren Studien (Hennemann et al. 2016) oftmals eine geringe Akzeptanz als maßgebliche Barriere für die Teilnahme an den digitalen Anwendungen (Hennemann et al. 2018). Das wohl einflussreichste theoretische Modell zur Erklärung des Zusammenhangs zwischen Akzeptanz und der Nutzung von Technologien ist das Technology Acceptance Model (TAM; Davis 1989). Es basiert auf der theoretischen Annahme der "theory of reasoned action" und postuliert, dass die tatsächliche Nutzung einer Technologie durch die Absicht, diese nutzen zu wollen, bestimmt wird (Ajzen und Fishbein 1980). Die Akzeptanz der Nutzenden wiederum wird durch die Einstellung gegenüber der Technologie beeinflusst (Davis 1989). In Arbeiten, die unterschiedliche Möglichkeiten zur Akzeptanzsteigerung erforschten, wurde festgestellt, dass Teilnehmende mit positiverer Einstellung eine höhere Akzeptanz gegenüber IBI aufwiesen (Casey et al. 2013; ApolinárioHagen et al. 2018; Donovan et al. 2015; Fleming und Merry 2013). Eine erhöhte Inanspruchnahme konnte ebenfalls nachgewiesen werden (Aerts und van Dam 2018).

Mithilfe sog. akzeptanzfördernder Interventionen („,acceptance facilitating intervention", AFI) werden potenzielle Nutzer*innen von IBI gezielt über die Wirksamkeit und den Nutzen der digitalen Gesundheitsanwendung informiert. Die Wirksamkeit von AFI konnte nachgewiesen werden, z. B. um Missverständnisse und Ängste abzubauen und die Nutzungsabsicht zu steigern (Lin et al. 2018; Gun et al. 2011; Mitchell und Gordon 2007). Auch eine positive Wirkung auf die Einstellung zu IBI konnte nachgewiesen werden (Casey et al. 2013). Selten wurde jedoch bislang untersucht, ob sich die Wirkung von AFI über die Akzeptanz hinaus auch auf die Adhärenz, also die regelmäßige Nutzung der Interventionen, auswirkt (Lin et al. 2018).

\section{Online-Selbsthilfeprogramm KEN-} Online

Das transdiagnostische psychodynamische Selbsthilfeprogramm KEN-Online setzt über 8 Einheiten hinweg den Fokus auf den Umgang mit Emotionen und deren Zusammenhang mit der zwischenmenschlichen Kommunikation (Becker et al. 2016). In einer randomisierten kontrollierten Feasibility-Studie wurde KENOnline hinsichtlich der Durchführbarkeit und Wirksamkeit als Nachsorgeintervention mit Patient*innen aus der stationären und teilstationären Psychosomatik untersucht (Becker et al. 2016). Die Intervention wies eine gute Akzeptanz auf; nur ca. $27 \%$ der Patient*innen, die über die Studie informiert wurden, lehnten die Teilnahme ab. Insgesamt 86\% (36/42) der nach Zufallsprinzip der Interventionsgruppe zugeordneten Teilnehmenden meldeten sich bei KEN-Online an. Im Verlauf der Untersuchung wurde ein stetiger Rückgang der Teilnahme verzeichnet. Circa 36\% (13/36) der Teilnehmenden nutzten KEN-Online aktiv und schlossen mindestens die Hälfte der Einheiten ab. Zwischen Interventions- und Wartelistenkontrollgruppe zeigten sich zum Ende der Intervention signifikante Unterschiede hinsichtlich der Einschätzung der Lebensqualität, der emotionalen Kompetenz sowie der depressiven Symptomatik, bei moderaten Effektgrößen. Die Wirksamkeit war größer unter Teilnehmenden, die das Programm vollständig abgeschlossen haben (Zwerenz et al. 2017). Integriert in die (teil-)stationäre Behandlung der Universitätsmedizin Mainz bearbeiten Patient*innen die edukativen Inhalte und können relevante Themenbereiche der Selbsthilfeintervention in ihre Therapiesitzungen einbringen.

\section{Fragestellungen}

Ziel der Studie ist es zu überprüfen, ob eine AFI die Nutzung einer IBI im Rah- 
men einer (teil-)stationären Psychotherapie fördert und den Zugang potenzieller Nutzer*innen zur IBI nachhaltig verbessert. Dazu soll der Einfluss einer AFI auf die Akzeptanz, die initiale Nutzung und die Adhärenz von KEN-Online untersucht werden (1). Des Weiteren wird überprüft, ob Patient*innen mit einer positiveren Einstellung gegenüber IBI eine höhere Akzeptanz für KEN-Online zeigen (2).

\section{Studiendesign und Unter- suchungsmethoden}

\section{Studiendesign}

Seit 2017 haben alle Patient*innen der Klinik und Poliklinik für Psychosomatische Medizin und Psychotherapie der Universitätsmedizin Mainz die Möglichkeit, KENOnline begleitend zur (teil-)stationären Behandlung zu nutzen. Pseudonymisierte Routinedaten bildeten die Datengrundlage der Untersuchung. Diese wurden retrospektiv für den Zeitraum Februar 2019 bis März 2020 ausgewertet. Zwischen September 2019 und März 2020 wurde zu Beginn der Behandlung eine AFI zu KEN-Online durchgeführt. Zur Ermittlung der Effekte der AFI wurden die Patient*innen im Rahmen eines Kohortendesigns in zwei Gruppen unterteilt. Alle Patient*innen, die zwischen Februar 2019 und August 2019 in der Klinik waren und sich für KEN-Online registrierten, wurden der Kontrollgruppe (KG) zugeordnet. Alle Patient*innen, die zwischen September 2019 und März 2020 in der Klinik waren und an der AFI teilgenommen hatten, wurden der Interventionsgruppe (IG) zugeordnet. Patient*innen der IG erhielten während ihres Aufenthalts zusätzlich zu den üblichen Informationen zu KEN-Online durch das Behandlungsteam und einen Flyer eine AFI, während Patient*innen der KG lediglich die Informationen durch das Behandlungsteam und einen Flyer zur KEN-Online erhielten. Patient*innen, die in diesem Zeitraum in der Klinik waren, jedoch nicht an der AFI teilgenommen hatten, wurden in den Analysen nicht berücksichtigt, um Selektionseffekte zu vermeiden.

\section{Akzeptanzfördernde Intervention}

Ziel der AFI war es, die Patient*innen standardisiert und strukturiert über KENOnline zu informieren und zur Teilnahme zu motivieren. Die Veranstaltung fand wöchentlich statt. Neu in die Klinik aufgenommene Patient ${ }^{*}$ innen erhielten eine Einladung. In der 30-minütigen PowerPoint-gestützten Veranstaltung stellte ein*e geschulte*r Psycholog*in Umfang, Inhalte und Ziele der Intervention vor und informierte über Wirksamkeit und Datensicherheit.

\section{Datenerhebung}

Grundlage bildete die Basisdokumentation der Klinik mit Informationen zu soziodemografischen Merkmalen, relevanten klinischen Konstrukten sowie Diagnosen nach ICD-10. Mithilfe von Selbstbeurteilungsfragebogen wurden Depressivität (Patient Health Questionnaire[PHQ]-9; Spitzer et al. 1999) und Ängstlichkeit (Generalized Anxiety Disorder[GAD]-7; Spitzer et al. 2006) erfasst. Zur Erhebung der Einstellung gegenüber IBI wurde im Rahmen der AFI der Selbstberichtsfragebogen Attitudes Towards Psychological Online Interventions Questionnaire (APOI; Schröder et al. 2017) eingesetzt.

Akzeptanz wurde entsprechend dem TAM als Absicht zur Inanspruchnahme der Intervention erfasst und durch die Registrierung bei KEN-Online operationalisiert. Die initiale Nutzung der Intervention wurde über den tatsächlichen Log-in bei KENOnline und die Adhärenz über die Anzahl der bearbeiteten Einheiten operationalisiert. Die entsprechenden objektiven Nutzungsdaten wurden über die individuellen Nutzungsstatistiken aus der KENOnline Datenbank entnommen und für die Datenauswertung aufbereitet.

\section{Datenauswertung}

In die Analysen gingen jeweils die Gesamtskalen ein. Skalensummenwerte wurden gebildet, sofern mindestens $80 \%$ der Items beantwortet wurden. Dabei wurden die Summenwerte auf Basis der Anzahl der vorliegenden Items gewichtet. Die Adhärenz wurde über die Anzahlen der Logins und abgeschlossenen Einheiten ope- rationalisiert. Darüber hinaus wurde die Anzahl der beendeten Einheiten dichotomisiert, und Nutzer*innen, die mehr als die Hälfte der Einheiten abgeschlossen haben, wurden als "completer" operationalisiert. Zur Prüfung von Unterschieden zwischen der IG und KG hinsichtlich der dichotomen Variablen Akzeptanz, initiale Nutzung und Completer-Status wurden $\chi^{2}$-Tests berechnet. Gruppenunterschiede in den kontinuierlichen Variablen zur Adhärenz wurden mit $t$-Tests für unabhängige Stichproben geprüft. Der Einfluss der Einstellung der Patient*innen gegenüber IBI auf die Akzeptanz wurde mithilfe der binär logistischen Regression überprüft. Neben der Einstellung wurden das Alter, das Geschlecht, die Angstsymptomatik und der Schulabschluss als unabhängige Variablen berücksichtigt. Die APOI-Werte wurden analog dem Vorgehen in anderen Studien (Baumeister et al. 2014; Ebert et al. 2015) nach Mittelwerten in 3 Gruppen kategorisiert, um geringe ( 1 bis 2,34$)$, moderate $(2,35$ bis 3,67$)$ und hohe $(3,68$ bis 5$)$ Ausprägungen zu beschreiben und die Häufigkeit der Kategorien zu berechnen.

\section{Ergebnisse}

\section{Stichprobenbeschreibung}

Während des untersuchten Zeitraums lagen die Daten von 231 Patient*innen vor. Es wurden 111 Patient*innen im teilstationären $(48,1 \%)$ und 120 im stationären Setting behandelt (51,9\%). Die Stichprobe setzte sich aus 130 Frauen und 101 Männern im Alter zwischen 19 und 76 Jahren zusammen (Mittelwert $[\mathrm{MW}]=38,94$, Standardabweichung [SD] $\pm 14,73$ ). Zwei Drittel der Patient*innen waren ledig und ein Viertel verheiratet. Ein Großteil der Patient*innen verfügte über das (Fach-)Abitur oder den Realschulabschluss. Am häufigsten waren affektive Störungen, gefolgt von Angst- und Persönlichkeitsstörungen vertreten. Im Mittel lagen 3 Diagnosen vor. Im Durchschnitt wiesen die behandelten Patient*innen entsprechend der Schweregradeinteilung des GAD-7 und PHQ-9 eine mittelgradige Angstsymptomatik sowie eine mittelgradige bis schwere depressive Symptomatikauf(- Tab. 1). Die Einstellung gegenüber $\mathrm{IBI}$ in der IG lag im Mittel bei 2,81 (SD $\pm 0,50$ ), was einer neutralen Ein- 
Tab. 1 Beschreibung der Stichprobenmerkmale

\begin{tabular}{|c|c|c|c|c|c|c|c|c|c|}
\hline \multirow{3}{*}{$\begin{array}{l}\text { Gruppe } \\
\text { Geschlecht }\end{array}$} & \multicolumn{3}{|c|}{ Gesamt } & \multicolumn{3}{|c|}{$\begin{array}{l}\text { Interventions- } \\
\text { gruppe }\end{array}$} & \multicolumn{3}{|c|}{ Kontrollgruppe } \\
\hline & $n$ & $\%$ & & $n$ & $\%$ & & $n$ & $\%$ & \\
\hline & \multicolumn{3}{|c|}{$(n=231)$} & \multicolumn{3}{|c|}{$(n=118)$} & \multicolumn{3}{|c|}{$(n=113)$} \\
\hline Weiblich & 130 & \multicolumn{2}{|l|}{56,3} & 69 & \multicolumn{2}{|l|}{58,5} & 61 & \multicolumn{2}{|l|}{54,0} \\
\hline Männlich & 101 & \multicolumn{2}{|l|}{43,7} & 49 & \multicolumn{2}{|l|}{41,5} & 52 & \multicolumn{2}{|l|}{46,0} \\
\hline Schulabschluss & \multicolumn{3}{|c|}{$(n=225)$} & \multicolumn{3}{|c|}{$(n=116)$} & \multicolumn{3}{|c|}{$(n=109)$} \\
\hline Noch in der Schule & 1 & \multicolumn{2}{|l|}{0,4} & 0 & \multicolumn{2}{|l|}{0,0} & 1 & \multicolumn{2}{|l|}{0,9} \\
\hline Kein Schulabschluss & 3 & \multicolumn{2}{|l|}{1,3} & 3 & \multicolumn{2}{|l|}{2,6} & 0 & \multicolumn{2}{|l|}{0,0} \\
\hline Hauptschulabschluss & 51 & \multicolumn{2}{|l|}{22,7} & 33 & \multicolumn{2}{|l|}{28,4} & 18 & \multicolumn{2}{|l|}{16,5} \\
\hline Realschulabschluss & 68 & \multicolumn{2}{|l|}{30,2} & 39 & \multicolumn{2}{|l|}{33,6} & 29 & 26,6 & \\
\hline (Fach-)Abitur & 86 & 38,2 & & 25 & 21,6 & & 61 & 56,0 & \\
\hline Andere & 16 & 7,1 & & 16 & 13,8 & & 0 & 0,0 & \\
\hline Familienstand & $(n=)$ & & & $(n=$ & & & $(n=$ & & \\
\hline Ledig & 149 & 65,1 & & 72 & 62,1 & & 77 & 68,1 & \\
\hline Verheiratet & 57 & 24,9 & & 30 & 25,9 & & 27 & 23,9 & \\
\hline $\begin{array}{l}\text { Geschieden, getrennt, } \\
\text { verwitwet }\end{array}$ & 23 & 10,0 & & 14 & 12,1 & & 9 & 8,0 & \\
\hline Berufstätigkeit & $(n=)$ & & & $(n=$ & & & $(n=$ & & \\
\hline Ja & 128 & 57,9 & & 65 & 57,5 & & 63 & 58,3 & \\
\hline Nein & 93 & 42,1 & & 48 & 42,5 & & 45 & 41,7 & \\
\hline Kliniksetting & $(n=)$ & & & $(n=$ & & & $(n=$ & & \\
\hline Teilstationär & 111 & 48,1 & & 64 & 41,6 & & 47 & 41,6 & \\
\hline Stationär & 120 & 51,9 & & 54 & 58,4 & & 66 & 58,4 & \\
\hline Psychische Störungen & $(n=)$ & & & $(n=$ & & & $(n=$ & & \\
\hline $\begin{array}{l}\text { Affektive Störung } \\
\text { (F30-F34) }\end{array}$ & 207 & 89,6 & & 106 & 89,8 & & 101 & 89,4 & \\
\hline $\begin{array}{l}\text { Angststörungen } \\
\text { (F40-F41) }\end{array}$ & 107 & 46,3 & & 60 & 50,8 & & 47 & 41,6 & \\
\hline $\begin{array}{l}\text { Persönlichkeits- } \\
\text { störungen (F60-F69) }\end{array}$ & 106 & 45,9 & & 52 & 44,1 & & 54 & 47,8 & \\
\hline $\begin{array}{l}\text { Depersonalisation-Dere- } \\
\text { alisation-Störung (F48.1) }\end{array}$ & 33 & 14,3 & & 22 & 18,6 & & 11 & 9,7 & \\
\hline $\begin{array}{l}\text { Somatoforme Störungen } \\
\text { (F45) }\end{array}$ & 70 & 30,3 & & 41 & 34,7 & & 29 & 25,7 & \\
\hline Andere Störungen & 137 & 59,3 & & 70 & 59,3 & & 67 & 59,3 & \\
\hline & $n$ & MW & \pm SD & $n$ & MW & SD & $n$ & MW & \pm SD \\
\hline Alter & 118 & 38,94 & $\pm 14,73$ & 113 & 36,65 & 13,69 & 113 & 41,32 & $\pm 15,45$ \\
\hline Anzahl psych. Diagnosen & 118 & 3,06 & $\pm 1,10$ & 113 & 3,18 & 0,99 & 113 & 2,93 & $\pm 1,19$ \\
\hline Ängstlichkeit (GAD-7) & 117 & 11,95 & $\pm 4,89$ & 112 & 12,59 & 4,90 & 112 & 11,28 & $\pm 4,82$ \\
\hline Depressivität (PHQ-9) & 117 & 14,95 & $\pm 5,85$ & 110 & 14,97 & 5,46 & 110 & 14,94 & $\pm 6,26$ \\
\hline Einstellung zur IBI (APOI) & 113 & 2,81 & $\pm 0,50$ & 113 & 2,81 & 0,50 & - & - & - \\
\hline
\end{tabular}

stellung entspricht. 23 Patient*innen wiesen eine negative $(20,4 \%), 89$ eine neutrale $(78,8 \%)$ und eine Patient*in eine positive Einstellung auf (0,9\%). Eine Übersicht der Stichprobenmerkmale kann - Tab. 1 entnommen werden.
Zur Überprüfung etwaiger Unterschiede zwischen der IG $(n=118)$ und der KG $(n=113)$ hinsichtlich soziodemografischer Variablen (Geschlecht, Alter, Berufstätigkeit) sowie psychischer Belastung (Depressivität, Ängstlichkeit) wurden $t$ Tests und $X^{2}$-Tests berechnet. Teilneh- mende der IG waren signifikant jünger $(t(229)=2,43, p=0,016, d=0,32)$ und wiesen eine signifikant erhöhte Angstsymptomatik $(t(227)=2,05, \quad p=0,041$, $d=0,27)$ auf.

\section{Akzeptanz}

Insgesamt registrierten sich $35,5 \%(n=82)$ der Gesamtstichprobe für KEN-Online. In der KG fragten $24,8 \%(n=28)$ der Patient*innen Zugangsdaten an und in der IG 45,8\% ( $n=54)$. Im $x^{2}$-Test zeigte sich ein signifikanter Unterschied in der Akzeptanz zwischen IG und KG $\left(X^{2}(1)=11,10, p<0,01\right.$, $\varphi=0,22)$.

\section{Initiale Nutzung}

Insgesamt loggten sich 75 Patient*innen $(32,5 \%)$ der Gesamtstichprobe bei KENOnline ein. Von den Patient*innen der KG loggten sich 23,0\% $(n=26)$, von den Patient*innen der IG 41,5\% $(n=49)$ ein. Ein $x^{2}$-Test ergab einen statistisch signifikanten Gruppenunterschied hinsichtlich der initialen Nutzung von KEN-Online $\left(X^{2}(1)=9,03, p<0,01, \varphi=0,20\right)$.

Sowohl die Akzeptanz- als auch die Aufnahmeraten sind in $\mathbf{0}$ Abb. 1 dargestellt.

\section{Adhärenz}

Hinsichtlich der Anzahl der abgeschlossenen Einheiten ergab sich kein signifikanter Unterschied $(t[80]=1,622 ; p=0,109)$ zwischen der $K G(M W=10,43 ; S D \pm 10,22)$ und der IG (MW=6,98; SD $\pm 8,52)$. Allerdings haben Teilnehmende der KG $(M W=2,43 ; S D \pm 2,60)$ signifikant mehr $(t[43,05]=2,318 ; p<0,05)$ Einheiten abgeschlossen als Teilnehmende der IG $(M W=1,12 ; S D \pm 1,97)$. In 0 Abb. 2 ist die Adhärenz über die Einheiten hinweg ersichtlich. Insgesamt 11 der Teilnehmenden, die sich für KEN-Online registrierten $(n=82)$, schlossen mehr als die Hälfte der Intervention ab (Completer). Das entspricht $13,4 \%$ der registrierten Teilnehmenden (IG: $11,1 \% ; n=5 ; \mathrm{KG}: 17,9 \%$; $n=6)$. $\operatorname{Im} \chi^{2}$-Test mit der dichotomisierten Adhärenzvariable (Completer vs. Noncompleter) unterschieden sich die beiden Gruppen nicht signifikant $\left(X^{2}(1)=0,72\right.$, $p=0,40, \varphi=-0,094)$. 


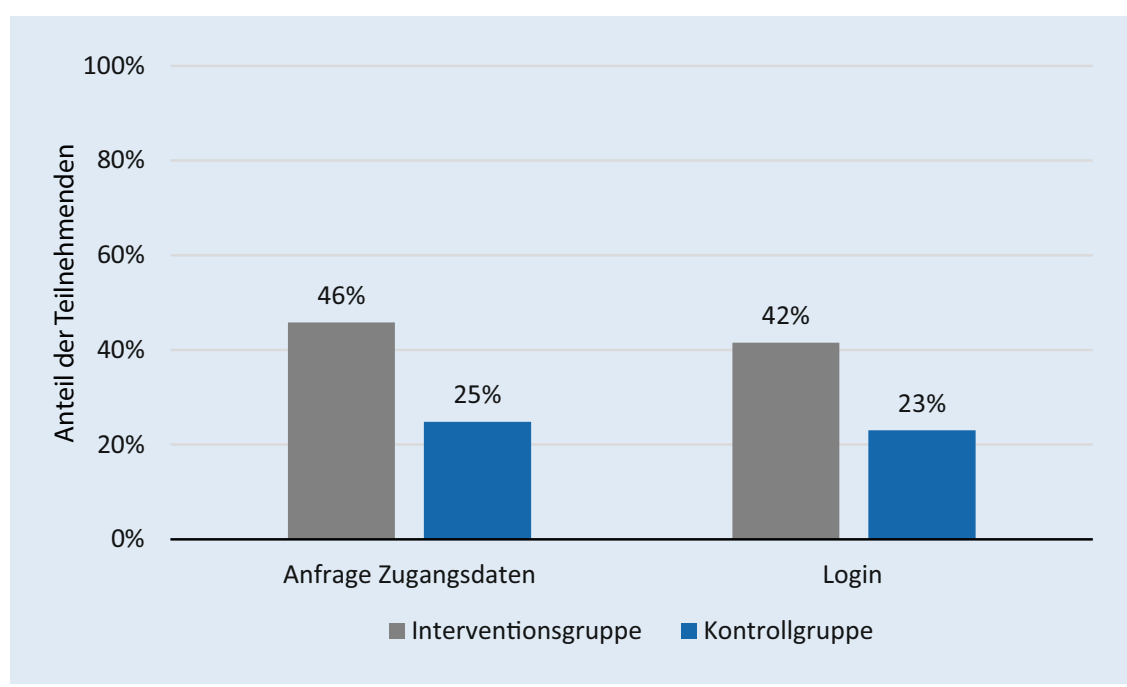

Abb. $1 \Delta$ Anteil der Interventions- $(n=118)$ und Kontrollgruppe $(n=113)$, die Zugangsdaten angefragt und sich bei KEN-Online angemeldet haben

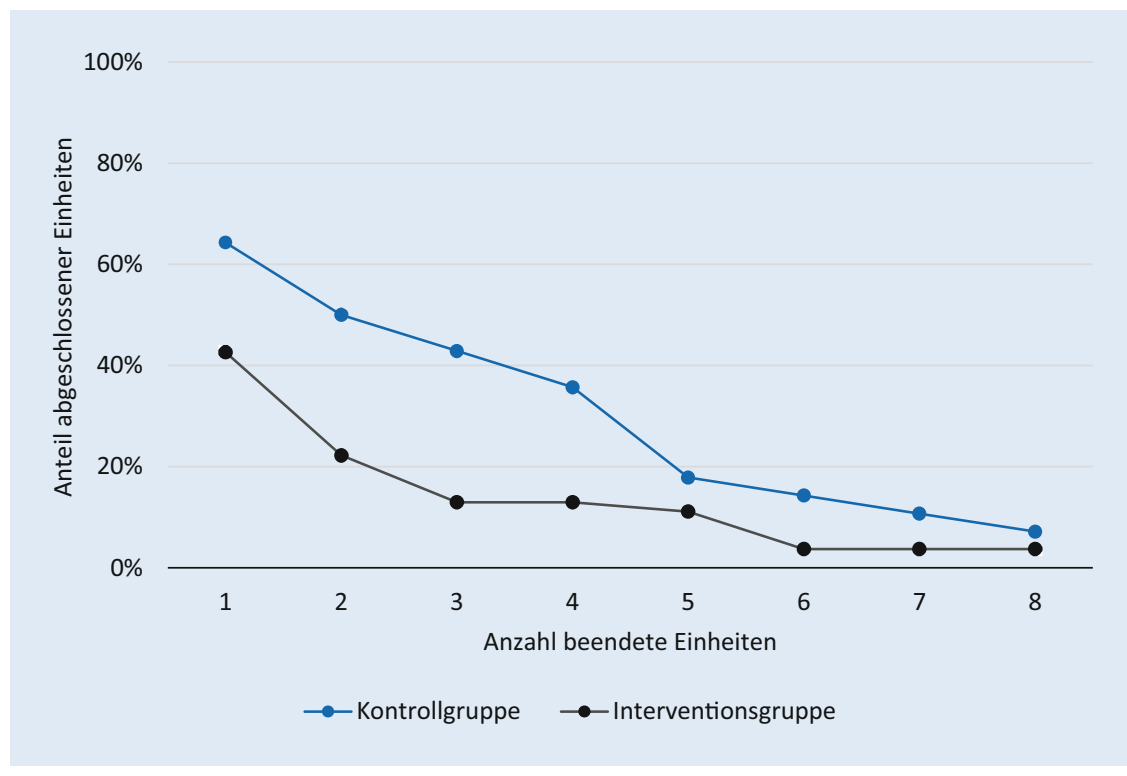

Abb. $2 \Delta$ Anteil der abgeschlossenen Einheiten in den beiden Gruppen

Um zu überprüfen, ob die Unterschiede bei Akzeptanz und Aufnahmerate zwischen IG und KG auf die AFI oder die signifikanten Unterschiede zwischen IG und KG zur Baseline bezüglich des Alters und der Angstsymptomatik zurückzuführen sind, wurden drei logistische Regressionen durchgeführt. Dabei wurde jeweils für den Einfluss von Alter und Angstsymptomatik kontrolliert. Passend zu den vorherigen Analysen zeigten sich signifikante Einflüsse der Gruppenzugehörigkeit auf die Akzeptanz (,odds ratio" $[\mathrm{OR}]=2,74$, Konfidenzintervall $[\mathrm{KI}]$ $\left.1,53-4,93, p=0,001, R^{2}=0,08\right)$ und die
Aufnahmerate $(\mathrm{OR}=2,52, \mathrm{KI}$ 1,40-4,56, $\left.p=0,002, R^{2}=0,07\right)$, nicht jedoch auf die Adhärenz (OR=0,64, KI 0,17-2,45, $\left.p=0,52, \mathrm{R}^{2}=0,04\right)$. Die Analysen zeigen darüber hinaus, abgesehen vom Alter bei der Akzeptanz (OR=1,02, KI 1,00-1,04, $p=0,049$ ), keinen signifikanten Einfluss des Alters und der Angstsymptomatik. Die Regression zeigt, dass die Akzeptanz mit jedem Lebensjahr um $2 \%$ steigt.

\section{Auswirkung der Einstellung}

Weder für die Einstellung noch für das Alter, das Geschlecht, die Angstsymptomatik und den Schulabschluss ergab sich ein signifikanter Haupteffekt. Patient*innen mit positiver Einstellung registrierten sich nicht signifikant häufiger für die Intervention $(\mathrm{OR}=1,02, \mathrm{KI} 0,97-1,08, p=0,36)$. Die aufgeklärte Varianz des Modells ist gering (Pseudo $R^{2}=0,05$ ).

\section{Diskussion}

\section{Ziel der Studie}

Bei der Implementierung von IBI spielen die Akzeptanz und Adhärenz eine wichtige Rolle, insbesondere da bekannt ist, dass die Wirksamkeit von IBI mit zunehmender Nutzungshäufigkeit ansteigt. Daher wurde im Rahmen einer Beobachtungsstudie im naturalistischen Setting untersucht, inwiefern eine AFI die Akzeptanz und Adhärenz von KEN-Online steigern kann. Darüber hinaus wurde der Zusammenhang zwischen der Einstellung gegenüber IBI sowie der Akzeptanz untersucht. Bei der AFI handelte es sich um eine niedrigschwellige Schulung in Form einer Informationsveranstaltung, bei der alle Patient*innen zu Behandlungsbeginn standardisiert und strukturiert über Ziele, Wirkweise und die technische Anwendung von KEN-Online informiert werden und die Möglichkeit haben, Fragen zu stellen.

\section{Interpretation der Ergebnisse und Vergleich mit der Literatur}

Mit dieser Untersuchung konnte gezeigt werden, dass die Akzeptanz und die initiale Nutzung von KEN-Online durch eine AFI um knapp $20 \%$ gesteigert werden können. Teilnehmende der AFI forderten signifikant häufiger Zugangsdaten zu KEN-Online an und nutzten KEN-Online initial signifikant häufiger als Patient*innen, die keine AFI erhielten. Diese Befunde decken sich mit Ergebnissen aus anderen Untersuchungen zur Wirkung von AFI (Apolinário-Hagen et al. 2018; Baumeister et al. 2014; Ebert et al. 2015; Casey et al. 2013). Darüber hinaus gab es einen geringen Alterseffekt in der Untersuchung. Mit zunehmendem Alter hat sich die Akzeptanz geringfügig verbessert. Dies widerspricht eigentlich der Annahme, dass jüngere Menschen offener gegenüber IBI eingestellt sind. Allerdings könnte der Unterschied auch auf die kli- 
nikspezifische Zusammensetzung der Patient*innengruppe zurückgeführt werden, da unter den jüngeren Patient ${ }^{*}$ innen deutlich häufiger solche mit Persönlichkeitsstörungen und niedrigerer Compliance für die Therapie zu finden sind.

In Bezug auf die Adhärenz von KENOnline fand sich ein kleiner signifikanter Unterschied dahingehend, dass Teilnehmende der KG etwas mehr Einheiten abgeschlossen haben als Teilnehmende der IG. Allerdings war der Anteil der Completer von KEN-Online mit 13,4\% insgesamt sehr gering und unterschied sich auch nicht zwischen den beiden Gruppen. Dies könnte daran liegen, dass die AFI vorwiegend auf die Förderung der Akzeptanz abzielte und nicht der Adhärenz. Zwar wird innerhalb der Veranstaltung betont, dass eine regelmäßige Nutzung und aktive Bearbeitung der Aufgaben und Übungen zur Wirksamkeit von KEN-Online beitragen, konkrete Hilfestellungen zur Etablierung einer regelmäßigen Nutzung werden jedoch nicht gegeben. Eine dahingehende Ergänzung der AFI könnte sich förderlich auf die Adhärenz auswirken. Eine weitere Erklärung könnte die sog. Intentions-Verhaltens-Lücke sein (Sheeran 2002), die die Diskrepanz zwischen der Akzeptanz und dem tatsächlichen Nutzungsverhalten beschreibt. Auch wenn Patient*innen angeben, eine bestimmte Verhaltensintention zu haben, stimmt diese nicht zwingend mit dem Zielverhalten überein. Dieses Phänomen wurde bereits in anderen Untersuchungen zu IBI beobachtet (Chiu und Eysenbach 2010; Lin et al. 2018).

Unabhängig von der Wirkung der AFI war die Adhärenz von KEN-Online deutlich geringer als in der randomisiert-kontrollierten Pilotstudie (Zwerenz et al. 2017). Dies könnte jedoch darauf zurückzuführen sein, dass die Teilnehmenden in der Pilotstudie eine therapeutische Unterstützung erhielten. Darüber hinaus ist jedoch auch bei anderen Interventionen eine höhere Drop-out-Rate bei der Implementierung von IBI in die Routineversorgung zu beobachten (Lin et al. 2018; Gilbody et al. 2015). Neben den spezifischen Eigenschaften von IBI spielen Kontextfaktoren, d.h. organisatorische und technische Voraussetzungen, im klinischen Setting eine wichtige Rolle für die Implementierung von IBI (Moessner und Bauer 2017). So kann beispielsweise die eingeschränkte Ausstattung mit WLAN auf den Stationen ein wichtiger Grund dafür sein, dass KEN-Online im Verlauf der (teil-)stationären Behandlung nicht so intensiv genutzt wurde, wie erwartet. Um $\mathrm{IBI}$ erfolgreich im klinischen Setting zu implementieren, sollte die technische Infrastruktur (WLAN, PC-Raum oder Tablets für Patient*innen) vorhanden sein, um die Nutzung möglichst niedrigschwellig zu ermöglichen.

Neben der Frage, inwieweit eine AFI die Akzeptanz und Adhärenz einer IBI erhöhen kann, wurde überprüft, ob die Einstellung gegenüber IBI einen Einfluss auf die Akzeptanz hat. So könnte die Erfassung der Einstellung gegenüber $\mid \mathrm{BI}$ beispielsweise dazu dienen, IBI gezielt Patient*innen mit positiver Einstellung anzubieten oder die Einstellung entsprechend durch eine AFI zu fördern, um in der Folge auch die Akzeptanz zu steigern. Ein solcher Zusammenhang zeigte sich jedoch entgegen vorheriger Forschungsergebnisse (Casey et al. 2013; Apolinário-Hagen et al. 2018; Donovan et al. 2015; Fleming und Merry 2013) nicht. Ein Grund für den fehlenden Zusammenhang zwischen Einstellung und Akzeptanz könnte darin begründet liegen, dass fast $80 \%$ der IG eine neutrale Einstellung berichteten, sodass die Varianz dieser Variable deutlich eingeschränkt und die Interpretation der Ergebnisse weniger gut möglich ist.

\section{Limitationen und Stärken der Studie}

Die Limitationen der Studie sind v. a. methodischer Natur. So erfolgte die Aufteilung in die IG und KG nicht per Randomisierung, sondern nach Zeiträumen, in denen die unterschiedlichen Studienbedingungen angeboten wurden, sodass auch andere Gründe (Kontext- und Zeitfaktoren), die nicht statistisch kontrolliert werden konnten, für die Unterschiede verantwortlich sein könnten. Darüber hinaus sind die Aussagen zur Adhärenz nur eingeschränkt zu interpretieren, da sich die Intervention vorwiegend auf die Akzeptanz konzentriert hat.

Eingeschränkt werden die Befunde zur Akzeptanz durch die Operationalisierung des Konstrukts mithilfe der Registrierung zur Online-Selbsthilfe. Die Schwierigkeit einer objektiven Messung der Akzeptanz wird durch die vielfältigen, häufig eigens entwickelten Bewertungsinstrumente unterschiedlicher Untersuchungen verdeutlicht (Baumeister et al. 2014; Chin et al. 2008). Die Verwendung von Likert-Skalen anstelle eines dichotomen Items könnte die Mehrdimensionalität des Konstrukts womöglich besser abbilden.

Eine Limitation besteht ebenso in dem nur sehr kleinen Anteil an Completern, d. h. Patient*innen, die KEN-Online mit mehr als der Hälfte der Einheiten genutzt haben. Der Großteil hat sich während der (teil-)stationären Behandlung gar nicht registriert oder nach erfolgreicher Registrierung nicht mehr eingeloggt bzw. das Programm abgebrochen. Hier könnte ein Grund darin liegen, dass Patient*innen zu Beginn ihrer Behandlung bereits mit dem vorhandenen F2F-Angebot gut ausgelastet sind und ein Online-Selbsthilfeprogramm, eher als „Add-on" und nicht als fester Behandlungsbaustein im Sinne eines „blended treatment" verstanden wird. Hierzu wäre eine bessere Verzahnung der beiden Behandlungsoptionen erforderlich (F2F und Online; Baumeister et al. 2018), was dann voraussichtlich auch zu einer höheren Adhärenz führen würde. Dies könnte in zukünftigen Studien durch eine Schulung und einen stärkeren Einbezug der Therapeut*innen verbessert werden. Darüber hinaus besteht hinsichtlich der Implementierung einer IBI in den klinischen Alltag eine maßgebliche Limitation darin, dass die technischen Voraussetzungen nicht optimal waren, um die IBI niedrigschwellig zu nutzen. So mussten Patient*innen auf private Endgeräte zurückgreifen. Hier besteht deutlicher Verbesserungsbedarf, wodurch die Akzeptanz und Adhärenz vermutlich gesteigert werden können.

Stärken der Studie sind die Auswertung von Routinedaten und die Untersuchung einer IBI im naturalistischen Design mit Patient*innen der Regelversorgung. Bisherige Untersuchungen wurden vorwiegend mit online rekrutierten Studienteilnehmenden oder unter kontrollierten Studienbedingungen durchgeführt. Durch eine solche Beobachtungsstudie wird die externe Validität erhöht, und somit können auch validere Aussagen über die Wirksamkeit von AFI in der klinischen Praxis getroffen werden. 
Weiterhin ist hervorzuheben, dass mit dieser Studie erste Erkenntnisse gesammelt wurden, wie die Akzeptanz und Nutzung einer psychodynamischen IBI verbessert werden kann. Da psychodynamische IBI bislang sowohl in der Forschung als auch in der Versorgung noch deutlich unterrepräsentiert sind (Eichenberg und Hübner 2018), besteht auf jeden Fall weiterer Forschungs- und Entwicklungsbedarf, um die Verfahrensvielfalt, die im F2F-Setting gegeben ist, auch im Rahmen der digitalen Gesundheitsversorgung abzudecken.

\section{Fazit für die Praxis}

- Die vorliegende Studie ist eine von wenigen Arbeiten, die in der Regelversorgung die Wirksamkeit einer angebotenen akzeptanzfördernden Intervention (AFI) für eine bereits evaluierte Intervention untersucht und den Zusammenhang zwischen der Einstellung gegenüber internetbasierten Interventionen (IBI) und deren Nutzung beleuchtet.

- Bei der Implementierung von IBI in die klinische Praxis kann die Förderung von Akzeptanz die initiale Nutzung von IBI verbessern. Allerdings sind weitere Maßnahmen erforderlich, um auch die Adhärenz nachhaltig zu fördern. Hier hat sich in verschiedenen Untersuchungen bereits gezeigt, dass insbesondere durch eine therapeutische Anleitung sowohl die Adhärenz als auch die Wirksamkeit von IBI gesteigert werden kann.

\section{Korrespondenzadresse}

\section{PD Dr. Rüdiger Zwerenz}

Klinik und Poliklinik für Psychosomatische Medizin und Psychotherapie, Universitätsmedizin der Johannes Gutenberg-Universität Untere Zahlbacher Str. 8, 55131 Mainz, Deutschland

ruediger.zwerenz@unimedizin-mainz.de

Funding. Open Access funding enabled and organized by Projekt DEAL.

\section{Einhaltung ethischer Richtlinien}

Interessenkonflikt. A. Kreis, J. Becker, M.E. Beutel und R. Zwerenz geben an, dass kein Interessenkonflikt besteht.

Für diesen Beitrag wurden von den Autoren keine Studien an Menschen oder Tieren durchgeführt.
Für die aufgeführten Studien gelten die jeweils dort angegebenen ethischen Richtlinien.

Open Access. Dieser Artikel wird unter der Creative Commons Namensnennung 4.0 International Lizenz veröffentlicht, welche die Nutzung, Vervielfältigung, Bearbeitung, Verbreitung und Wiedergabe in jeglichem Medium und Format erlaubt, sofern Sie den/die ursprünglichen Autor(en) und die Quelle ordnungsgemäß nennen, einen Link zur Creative Commons Lizenz beifügen und angeben, ob Änderungen vorgenommen wurden.

Die in diesem Artikel enthaltenen Bilder und sonstiges Drittmaterial unterliegen ebenfalls der genannten Creative Commons Lizenz, sofern sich aus der Abbildungslegende nichts anderes ergibt. Sofern das betreffende Material nicht unter der genannten Creative Commons Lizenz steht und die betreffende Handlung nicht nach gesetzlichen Vorschriften erlaubt ist, ist für die oben aufgeführten Weiterverwendungen des Materials die Einwilligung des jeweiligen Rechteinhabers einzuholen.

Weitere Details zur Lizenz entnehmen Sie bitte der Lizenzinformation auf http://creativecommons.org/ licenses/by/4.0/deed.de.

\section{Literatur}

Aerts JEM, van Dam A (2018) Blended e-health in cognitive behavioural therapy: usage intensity, attitude and therapeutic alliance in clinical practice. Psychology 09:2422-2435

Ajzen I, Fishbein M (1980) Understanding attitudes and predicting social behavior. Prentice-Hall, Englewood Cliffs

Andersson G, Carlbring P, Titov N, Lindefors N (2019) Internet interventions for adults with anxiety and mood disorders: a narrative umbrella review of recent meta-analyses. Can J Psychiatry 64:465-470

Andersson G, Cuijpers P, Carlbring P, Riper H, Hedman E (2014) Guided Internet-based vs. face-to-face cognitive behavior therapy for psychiatric and somatic disorders: a systematic review and metaanalysis. World Psychiatry 13:288-295

Apolinário-Hagen J, Harrer M, Kählke F, Fritsche L, Salewski C, EbertDD (2018) Public attitudes toward guided Internet-based therapies: web-based survey study. JMIR Ment Health 5(2):e10735. https://doi.org/10.2196/10735

Baumeister H, Grässle C, Ebert DD, Krämer LV (2018) Blended Psychotherapy - verzahnte Psychotherapie: Das Beste aus zwei Welten? Psychother Dialog 19:33-38. https://doi.org/10. 1055/a-0592-0264

Baumeister H, Nowoczin L, Lin J, Seifferth H, Seufert J, Laubner K, Ebert DD (2014) Impact of an acceptance facilitating intervention on diabetes patients' acceptance of Internet-based interventions for depression: a randomized controlled trial. Diabetes Res Clin Pract 105:30-39

Becker J, Zwerenz R, Johansson R, Frederick RJ, Andersson G, Beutel M (2016) Using a transdiagnostic, psychodynamic online self-help intervention to maintain inpatient psychosomatic treatment effects: Study protocol of a feasibility study. Internet Interv 5:30-35

Casey LM, Joy A, Clough BA (2013) The impact of information on attitudes toward e-mental health services. Cyberpsychol Behav Soc Netw 16:593-598

Chin, Johnson, Schwarz (2008) A fast form approach to measuring technology acceptance and other constructs. MISQ 32:687. https://doi.org/10. 2307/25148867

Chiu TML, Eysenbach G (2010) Stages of use: consideration, initiation, utilization, and outcomes of an internet-mediated intervention. BMC medical informatics and decision making 10:73. https:// doi.org/10.1186/1472-6947-10-73

Davis FD (1989) Perceived usefulness, perceived ease of use, and user acceptance of information technology. MISQ 13:319

Donovan CL, Poole C, Boyes N, Redgate J, March S (2015) Australian mental health worker attitudes towards CCBT: What is the role of knowledge? Are there differences? Can we change them? Internet Interv 2:372-381

Ebert DD, Berking $M$, Cuijpers $P$, Lehr D, Pörtner $M$, Baumeister $\mathrm{H}$ (2015) Increasing the acceptance of internet-based mental health interventions in primarycare patients with depressivesymptoms. A randomized controlled trial. J Affect Disord 176:9-17

Eichenberg C, Hübner L (2018) Psychoanalyse via Internet. Psychotherapeut 63:283-290

Fleming T, Merry S (2013) Youth work service providers' attitudes towards computerized CBT for adolescents. Behav Cogn Psychother 41:265-279

Gieselmann A, Böckermann M, Pietrowsky R (2015) Internetbasierte Gesundheitsinterventionen. Psychotherapeut 60:433-440

Gilbody S, Littlewood E, Hewitt C, Brierley G, Tharmanathan P, Araya R, Barkham M, Bower P, Cooper C, Gask L, Kessler D, Lester H, Lovell K, Parry G, Richards DA, Andersen P, Brabyn S, Knowles S, Shepherd C, Tallon D, White D (2015) Computerised cognitive behaviour therapy (cCBT) as treatment for depression in primary care (REEACT trial): large scale pragmatic randomised controlled trial. BMJ351:h5627

Gun SY, Titov N, Andrews G (2011) Acceptability of Internet treatment of anxiety and depression. Australas Psychiatry 19:259-264

Hedman E, Ljótsson B, Lindefors N (2012) Cognitive behavior therapy via the Internet: a systematic review of applications, clinical efficacy and costeffectiveness. Expert Rev Pharmacoeconom Outcomes Res 12:745-764. https://doi.org/10. 1586/erp. 12.67

Hennemann S, Beutel M, Zwerenz R (2016) Drivers and barriers to acceptance of web-based aftercare of patients in inpatient routine care: a crosssectional survey. J Med Internet Res 18:e337. https://doi.org/10.2196/jmir.6003

Hennemann S, Witthöft M, Bethge M, Spanier K, Beutel M, Zwerenz R (2018) Acceptance and barriers to access of occupational e-mental health: cross-sectional findings from a healthrisk population of employees. Int Arch Occup Environ Health 91:305-316

Jacobi F, Höfler M, Strehle J, Mack S, Gerschler A, Scholl L, Busch MA, Maske U, Hapke U, Gaebel W, Maier W, Wagner M, Zielasek J, Wittchen H-U (2014) Psychische Störungen in der Allgemeinbevölkerung; Studie zur Gesundheit Erwachsener in Deutschland und ihr Zusatzmodul Psychische Gesundheit (DEGS1MH). Nervenarzt 85:77-87

Lin J, Ebert DD, Lehr D, Berking M, Baumeister H (2013) Internetbasierte kognitiv-behaviorale Behandlungs-ansätze: State of the Art und 
Einsatzmöglichkeiten in der Rehabilitation. Rehabilitation 52:155-163

Lin J, Faust B, Ebert DD, Krämer L, Baumeister H (2018) A web-based acceptance-facilitating intervention for identifying patients' acceptance, uptake, and adherence of Internet- and mobile-based pain interventions: randomized controlled trial.J Med Internet Res 20:e244

Mitchell N, Gordon PK (2007) Attitudes towards computerized CBT for depression amongst a student population. Behav Cogn Psychother 35:421-430

Moessner M, Bauer S (2017) E-Mental-Health und internetbasierte Psychotherapie. Psychotherapeut 62:251-266

Schröder J, Berger T, Meyer B, Lutz W, Hautzinger M, Späth C, Eichenberg C, Klein JP, Moritz S (2017) Attitudes towards Internet interventions among psychotherapists and individuals with mild to moderate depression symptoms. Cogn Ther Res 41:745-756

Sheeran P (2002) Intention—behavior relations: a conceptual and empirical review. Eur Rev Soc Psychol 12:1-36

Spitzer RL, Kroenke K, Williams JB (1999) Validation and utility of a self-report version of PRIMEMD: the PHQ primary care study. Primary care evaluation of mental disorders. Patient health questionnaire. JAMA 282:1737-1744

Spitzer RL, Kroenke K, Williams JBW, Löwe B (2006) A brief measure for assessing generalized anxiety disorder: the GAD-7. Arch Intern Med 166:1092-1097

Walker ER, McGee RE, Druss BG (2015) Mortality in mental disorders and global disease burden implications: a systematic review and metaanalysis. JAMA Psychiatry 72:334-341

Zwerenz R, Beutel M (2017) Online-Interventionen zur Behandlung psychischer Erkrankungen und Belastungen. Welche aktuellen Ansätze gibt es und wie wirksam sind diese? Arbeitsmed Sozialmed Umweltmed 52:452-460

Zwerenz R, Becker J, Johansson R, Frederick RJ, Andersson G, Beutel ME (2017) Transdiagnostic, psychodynamic web-based self-help intervention following inpatient psychotherapy: results of a feasibility study and randomized controlled trial. JMIR Ment Health 4:e41
Influence of acceptance-facilitating interventions on the use of an online self-help. Naturalistic investigation in day clinic and inpatient psychosomatic psychotherapy

Background: Despite increasing empirical evidence for the effectiveness in the treatment of mental disorders, internet-based interventions (IBI) are still confronted by low acceptance and high dropout rates. Acceptance-facilitating interventions (AFI) represent a promising measure to improve acceptance of possible users. The effectiveness of AFI on adherence, which has a substantial influence on the effectiveness of $|B|$, has so far been examined in only a few empirical investigations. Objective: The aim of this study was to examine the influence of an AFI on the acceptance, initial use, and adherence of the transdiagnostic psychodynamic online self-help program that aims to use the power of one's own emotions and is called "Die Kraft der eigenen Emotionen nutzen, (KEN-Online)".

Material and methods: Routine data from 231 patients were analyzed, who were offered the option of using KEN-Online during a day clinic and inpatient treatment within the framework of an AFI integrated into the routine setting. In the cohort design patients who participated in an AFI were compared with a historical control group of patients who did not receive this offer. Furthermore, the relationship between the attitude and the acceptance of the program was examined in the study group (with AFI).

Results: Both acceptance and the initial use of KEN-Online were almost $20 \%$ higher ( $p<0.01$ each) among participants of the AFI group compared to the control group. Concerning the adherence of $K E N$-Online, a small difference was observed in that participants in the control group completed significantly $(p<0.05)$ more units than participants in the intervention group; however, the overall percentage of completers of KEN-Online was very low with $13.4 \%$. No evidence was found for an impact of the attitude on acceptance.

Conclusion: The present study showed that AFI can help facilitate acceptance and initial use of IBI by supplying patients in routine care with standardized and structured information about the intervention; however, regarding the promotion of adherence, additional measures are required.

\section{Keywords}

Internet-based intervention $\cdot$ Acceptance $\cdot$ Treatment adherence $\cdot$ Attitude $\cdot$ Health care utilization 